\title{
TECHNICALITIES IN THE CALCULATION OF THE 3RD POST-NEWTONIAN DYNAMICS
}

\author{
PIOTR JARANOWSKI \\ Max-Planck-Society, Research Unit "Theory of Gravitation" \\ at the Friedrich-Schiller-University, 07743 Jena, Germany \\ E-mail: pio@gravi.physik.uni-jena.de \\ (Permanent address: Institute of Physics, Warsaw University Branch \\ Lipowa 41, 15-424 Biatystok, Poland \\ E-mail:pijar@cksr.ac.bialystok.pl)
}

\begin{abstract}
Dynamics of a point-particle system interacting gravitationally according to the general theory of relativity can be analyzed within the canonical formalism of Arnowitt, Deser, and Misner. To describe the property of being a point particle one can employ Dirac delta distribution in the energy-momentum tensor of the system. We report some mathematical difficulties which arise in deriving the 3rd post-Newtonian Hamilton's function for such a system. We also offer ways to overcome partially these difficulties.
\end{abstract}

1. Introduction. Dynamics of a point-particle system interacting gravitationally according to the theory of general relativity can be studied within the canonical formalism devised by Arnowitt, Deser, and Misner [1]. In the framework of this formalism an approximation scheme for solving equations of motion was developed (for more details see lecture of Schäfer in this volume). The ultimate goal at each level of approximation is to calculate the Hamilton's function of the system. In this paper we report some mathematical difficulties which arise in the calculation of the 3rd post-Newtonian Hamilton's function. The source of the difficulties is the presence of Dirac delta distributions in the energy-momentum tensor describing the system of point particles. Similar problems are already present in the calculation of lower order Hamilton's functions, but it seems that at these orders the methods used to overcome them do not give any ambiguities in the final results, even if the calculations are performed to some extent in a "naive" way.

Up to now the calculations of the Hamilton's function for a point-particle system was unambigously done up to 2.5 post-Newtonian order [8] and also for 3.5 order [3]. For these

1991 Mathematics Subject Classification: 83C10, 83C25.

Research partially supported by KBN grant 2 P303D 02111.

The paper is in final form and no version of it will be published elsewhere. 
orders there exist several independent derivations of the equations of motion. All these derivations were found to be compatible with each other. Jaranowski and Schäfer found recently [4], that at the 3rd post-Newtonian order the methods presented in this paper are sufficient to calculate the 3rd post-Newtonian Hamilton's function almost unambigously. There exist a few terms, for which consistency may not be achievable. More precisely, via integration by parts, these ambiguous terms can be represented in two different but equivalent ways. The regularization methods applied to both representations give different results but the difference does not depend on which regularization method is used.

The plan of the paper is as follows. Use of the Dirac delta distributions causes a necessity to devise a rule to give meaning to integrals of the form $\int d^{3} x f(\boldsymbol{x}) \delta(\boldsymbol{x})$, where the function $f$ is assumed to be singular at $\boldsymbol{x}=0$. Such a rule is defined in Section 2 . Another difficulty lies in the necessity of regularizing a class of divergent integrals with nonintegrabilities of the rational type. Section 3 is devoted to the problem of regularizing integrals of some specific type. Section 4 shows how to properly differentiate homogeneous and locally nonintegrable functions under the integral sign. In the Section 5 we study the problem of compatibility of different regularization techniques. And finally, Section 6 suggests a regularization procedure for more general integrals.

We employ the following notation. All functions studied here are real valued functions defined on the 3 -dimensional Euclidean space $\mathbb{R}^{3}$ (possibly with some isolated points removed), $\mathbb{R}^{3}$ is endowed with a standard metric and a scalar product, denoted by a dot. Letters $a, b, \ldots$ are particles labels, so $\boldsymbol{x}_{a} \in \mathbb{R}^{3}$ denotes the position of the $a$ th particle. We also define: $\boldsymbol{r}_{a}:=\boldsymbol{x}-\boldsymbol{x}_{a}, r_{a}:=\left|\boldsymbol{r}_{a}\right|, \boldsymbol{n}_{a}:=\boldsymbol{r}_{a} / r_{a}$; and for $a \neq b: \boldsymbol{r}_{a b}:=\boldsymbol{x}_{a}-\boldsymbol{x}_{b}$, $r_{a b}:=\left|\boldsymbol{r}_{a b}\right|, \boldsymbol{n}_{a b}:=\boldsymbol{r}_{a b} / r_{a b} ;|\cdot|$ stands here for the length of a vector.

2. Hadamard's "partie finie" regularization. The procedure described here was used by Schäfer (cf. Appendix B in [8]) in the calculations of the 2nd and 2.5 postNewtonian Hamilton's functions for a point-particle system.

Let $f$ be a real valued function defined in a neighbourhood of a point $\boldsymbol{x}_{o} \in \mathbb{R}^{3}$, excluding this point. At $\boldsymbol{x}_{o}$ the function $f$ is assumed to be singular. We define the family of auxiliary complex valued functions $f_{\boldsymbol{n}}$ (labelled by unit vectors $\boldsymbol{n}$ ) in the following way:

$$
f_{\boldsymbol{n}}: \mathbb{C} \ni \varepsilon \mapsto f_{\boldsymbol{n}}(\varepsilon):=f\left(\boldsymbol{x}_{o}+\varepsilon \boldsymbol{n}\right) \in \mathbb{C} .
$$

We expand $\boldsymbol{f}_{\boldsymbol{n}}$ into Laurent series around $\varepsilon=0$ :

$$
f_{\boldsymbol{n}}(\varepsilon)=\sum_{m=-N}^{\infty} a_{m}(\boldsymbol{n}) \varepsilon^{m}
$$

Coefficients $a_{m}$ of this expansion depend on the unit vector $\boldsymbol{n}$. We define the regularized value of the function $f$ at $\boldsymbol{x}_{o}$ as the coefficient at $\varepsilon^{0}$ in the expansion (2) averaged over all unit vectors $\boldsymbol{n}$ :

$$
f_{\mathrm{reg}}\left(\boldsymbol{x}_{o}\right):=\frac{1}{4 \pi} \oint d \Omega a_{0}(\boldsymbol{n}) .
$$

We use formula (3) to compute all integrals which contain Dirac delta distribution. 
It means that we define

$$
\int d^{3} x f(\boldsymbol{x}) \delta\left(\boldsymbol{x}-\boldsymbol{x}_{a}\right):=f_{\text {reg }}\left(\boldsymbol{x}_{a}\right) .
$$

EXAMPle 2.1. Let us consider the integral $\int d^{3} x \delta\left(\boldsymbol{x}-\boldsymbol{x}_{a}\right) /\left(r_{a}^{2} r_{b}^{2}\right)$, for $a \neq b$. Here the function $f_{\boldsymbol{n}}$ is equal to

$$
f_{\boldsymbol{n}}(\varepsilon)=\frac{1}{\varepsilon^{2}\left|\boldsymbol{r}_{a b}+\varepsilon \boldsymbol{n}\right|^{2}} .
$$

The expansion of $f_{\boldsymbol{n}}$ into Laurent series, up to $\varepsilon^{0}$, is given by

$$
f_{\boldsymbol{n}}(\varepsilon)=\frac{1}{r_{a b}^{2}} \frac{1}{\varepsilon^{2}}-\frac{2\left(\boldsymbol{n} \cdot \boldsymbol{r}_{a b}\right)}{r_{a b}^{4}} \frac{1}{\varepsilon}+\left(\frac{4\left(\boldsymbol{n} \cdot \boldsymbol{r}_{a b}\right)^{2}}{r_{a b}^{6}}-\frac{1}{r_{a b}^{4}}\right)+\mathcal{O}(\varepsilon) .
$$

From (3) and (4) we have

$$
\int d^{3} x \frac{1}{r_{a}^{2} r_{b}^{2}} \delta\left(\boldsymbol{x}-\boldsymbol{x}_{a}\right)=\frac{1}{4 \pi} \oint d \Omega\left(\frac{4\left(\boldsymbol{n} \cdot \boldsymbol{r}_{a b}\right)^{2}}{r_{a b}^{6}}-\frac{1}{r_{a b}^{4}}\right)=\frac{1}{3 r_{a b}^{4}},
$$

where we have taken into account that

$$
\frac{1}{4 \pi} \oint d \Omega\left(\boldsymbol{n} \cdot \boldsymbol{r}_{a b}\right)^{2}=\frac{1}{3} r_{a b}^{2}
$$

We can also use definition (3) to calculate some divergent integrals as limiting values of integrals, which are convergent.

EXAMPLE 2.2. Let us take the following integral, which is convergent for $b \neq a$ and $c \neq a, b$ (cf. equation (A3.3) in Appendix 3 of [6]):

$$
\int d^{3} x \frac{n_{a}^{i} n_{b}^{j}}{r_{a}^{2} r_{b}^{2} r_{c}}=4 \pi\left[-\frac{\left(n_{a b}^{i}-n_{c a}^{i}\right)\left(n_{a b}^{j}-n_{b c}^{j}\right)}{\left(r_{a b}+r_{b c}+r_{c a}\right)^{2}}+\frac{\delta_{i j}-n_{a b}^{i} n_{a b}^{j}}{r_{a b}\left(r_{a b}+r_{b c}+r_{c a}\right)}\right] .
$$

Let us now consider the divergent integral (here $a \neq c$ )

$$
\int d^{3} x \frac{n_{a}^{i} n_{a}^{j}}{r_{a}^{4} r_{c}}
$$

which can be obtained from the left-hand side of (9) by identifying $b$ with $a$. Let us define the function $f$ of the variable $\boldsymbol{x}_{c}$ such that $f\left(\boldsymbol{x}_{c}\right)$ is equal to the right-hand side of (9). The function $f$ is singular at $\boldsymbol{x}_{c}=\boldsymbol{x}_{a}$. Then we define:

$$
\left[\int d^{3} x \frac{n_{a}^{i} n_{a}^{j}}{r_{a}^{4} r_{c}}\right]_{\mathrm{reg}}:=f_{\mathrm{reg}}\left(\boldsymbol{x}_{a}\right)
$$

Using definition (3) we obtain:

$$
\left[\int d^{3} x \frac{n_{a}^{i} n_{a}^{j}}{r_{a}^{4} r_{c}}\right]_{\mathrm{reg}}=\frac{\pi}{r_{a c}^{2}}\left(n_{a c}^{i} n_{a c}^{j}-\delta_{i j}\right)
$$

3. Riesz's formula for volume integrals. The following formula, firstly derived by Riesz (see equations (7) and (10) in Chapter 2 of [7]), can serve as a tool to regularize a 
class of divergent integrals of $r_{a}^{\alpha} r_{b}^{\beta}$ (for $a \neq b$ ) using the analytic continuation arguments:

$$
\begin{aligned}
{\left[\int d^{3} x r_{a}^{\alpha} r_{b}^{\beta}\right]_{\mathrm{reg}} } & :=R(\alpha, \beta) r_{a b}^{\alpha+\beta+3}, \\
R(\alpha, \beta) & :=\pi^{3 / 2} \frac{\Gamma\left(\frac{\alpha+3}{2}\right) \Gamma\left(\frac{\beta+3}{2}\right) \Gamma\left(-\frac{\alpha+\beta+3}{2}\right)}{\Gamma\left(-\frac{\alpha}{2}\right) \Gamma\left(-\frac{\beta}{2}\right) \Gamma\left(\frac{\alpha+\beta+6}{2}\right)} .
\end{aligned}
$$

Of course definition (13) can be directly used only for those numbers $\alpha$ and $\beta$ for which $R(\alpha, \beta)$ exists.

ExAmPLE 3.1. One can use (13) to calculate the value of the following convergent integral (here $a \neq b)$

$$
\int d^{3} x \frac{1}{r_{a}^{2} r_{b}^{2}}=\frac{\pi^{3}}{r_{a b}} .
$$

EXAMPLE 3.2. The integral of $1 /\left(r_{a}^{4} r_{b}\right), a \neq b$, is divergent because the integrand has the nonintegrable singularity at $\boldsymbol{x}=\boldsymbol{x}_{a}$. From (13) one obtains the regularized value of this integral

$$
\left[\int d^{3} x \frac{1}{r_{a}^{4} r_{b}}\right]_{\mathrm{reg}}=-\frac{2 \pi}{r_{a b}^{2}} .
$$

EXAMPLE 3.3. The integral of $1 /\left(r_{a} r_{b}\right), a \neq b$, is divergent because the integrand falls off too slowly at infinity. Its regularized value, which follows from (13), is

$$
\left[\int d^{3} x \frac{1}{r_{a} r_{b}}\right]_{\mathrm{reg}}=-2 \pi r_{a b} .
$$

We need a regularization procedure which is more general than that defined by (13). Let $R(\alpha, \beta)$ does not exist for some $\alpha$ and $\beta$, so the rule (13) is not directly applicable. Then we define the auxiliary function

$$
I_{\varepsilon}^{\mu, \nu}(\alpha, \beta):=R(\alpha+\mu \varepsilon, \beta+\nu \varepsilon) r_{a b}^{\alpha+\beta+(\mu+\nu) \varepsilon+3}
$$

and study the limit

$$
\lim _{\varepsilon \rightarrow 0} I_{\varepsilon}^{\mu, \nu}(\alpha, \beta) .
$$

In general the limit (16) will depend on $\mu$ and $\nu$. In the calculation of the 3rd postNewtonian Hamilton's function for a point-particle system it is enough to consider only limits, which depend linearly on the fraction $\mu / \nu$ or $\nu / \mu$, or both [4], so the most general form of such a limit is

$$
\lim _{\varepsilon \rightarrow 0} I_{\varepsilon}^{\mu, \nu}(\alpha, \beta)=A(\alpha, \beta)+B(\alpha, \beta) \frac{\mu}{\nu}+C(\alpha, \beta) \frac{\nu}{\mu},
$$

where $A, B, C$ do not depend on $\mu$ and $\nu$. As the regularized value of the integral of $r_{a}^{\alpha} r_{b}^{\beta}$ we take the number $A(\alpha, \beta)$, i.e. this part of the limit (16) which does not depend on the direction of approaching the point $(\alpha, \beta) \in \mathbb{R}^{2}$ in the limiting process. Thus we define

$$
\left[\int d^{3} x r_{a}^{\alpha} r_{b}^{\beta}\right]_{\mathrm{reg}}:=A(\alpha, \beta) .
$$


EXAMPLE 3.4. The integral of $1 /\left(r_{a}^{5} r_{b}\right)$ can not be computed directly from definition (13), because for $\alpha=-5$ and $\beta=-1$ formula (14) is singular. To regularize this integral we use definition (18). The result is

$$
\lim _{\varepsilon \rightarrow 0} I_{\varepsilon}^{\mu, \nu}(-5,-1)=-\frac{2}{3}\left(1+\frac{\nu}{\mu}\right) \frac{\pi}{r_{a b}^{3}}, \quad\left[\int d^{3} x \frac{1}{r_{a}^{5} r_{b}}\right]_{\mathrm{reg}}=-\frac{2 \pi}{3 r_{a b}^{3}} .
$$

It turns out that not for all $\alpha$ and $\beta$ the integrals of $r_{a}^{\alpha} r_{b}^{\beta}$ can be regularized by means of the rule (18), but we can always identify some linear combinations of the integrals, which are regularizable with the aid of a generalization of formula (18). Let us consider such a combination of integrands

$$
\sum_{i=1}^{N} c_{i} r_{a}^{\alpha_{i}} r_{b}^{\beta_{i}}
$$

where $c_{i}, \alpha_{i}$, and $\beta_{i}$ are constants. For the combination we calculate the limit

$$
\lim _{\varepsilon \rightarrow 0} \sum_{i=1}^{N} c_{i} I_{\varepsilon}^{\mu, \nu}\left(\alpha_{i}, \beta_{i}\right) .
$$

It is again enough to restrict to limits which are of the form (17)

$$
\lim _{\varepsilon \rightarrow 0} \sum_{i=1}^{N} c_{i} I_{\varepsilon}^{\mu, \nu}\left(\alpha_{i}, \beta_{i}\right)=A+B \frac{\mu}{\nu}+C \frac{\nu}{\mu} .
$$

The regularized value of the integral of the combination (19) is defined as:

$$
\left[\int d^{3} x \sum_{i=1}^{N} c_{i} r_{a}^{\alpha_{i}} r_{b}^{\beta_{i}}\right]_{\mathrm{reg}}:=A .
$$

EXAMPLE 3.5. Let us consider the three integrands $(a \neq b): 1 /\left(r_{a} r_{b}^{3}\right), 1 /\left(r_{a}^{3} r_{b}\right)$, and $1 /\left(r_{a}^{3} r_{b}^{3}\right)$. Integrals of any of them can not, by means of (13) or (18), be regularized separately. But we can apply definition (22) to the following combination

The result reads

$$
\frac{1}{r_{a} r_{b}^{3}}+\frac{1}{r_{a}^{3} r_{b}}-\frac{r_{a b}^{2}}{r_{a}^{3} r_{b}^{3}} \text {. }
$$

$$
\begin{aligned}
\lim _{\varepsilon \rightarrow 0}\left[I_{\varepsilon}^{\mu, \nu}(-1,-3)+I_{\varepsilon}^{\mu, \nu}(-3,-1)-r_{a b}^{2} I_{\varepsilon}^{\mu, \nu}(-3,-3)\right] & =\frac{8 \pi}{r_{a b}}, \\
& {\left[\int d^{3} x\left(\frac{1}{r_{a} r_{b}^{3}}+\frac{1}{r_{a}^{3} r_{b}}-\frac{r_{a b}^{2}}{r_{a}^{3} r_{b}^{3}}\right)\right]_{\mathrm{reg}}=\frac{8 \pi}{r_{a b}} . }
\end{aligned}
$$

Here the numbers $B$ and $C$ from (21) are equal to zero.

4. The rule to differentiate homogeneous functions. In our calculations we have to differentiate some homogeneous and locally nonintegrable functions under the integral sign. To do this properly we must use an enhanced rule of differentiation, which comes from distribution theory [2]. The importance of this rule will be illustrated in the next section. 
Let $f$ be a real valued function defined in a neighbourhood of the origin in $\mathbb{R}^{3} . f$ is said to be a positively homogeneous function of degree $\lambda$, if for any number $a>0$

$$
f(a \boldsymbol{x})=a^{\lambda} f(\boldsymbol{x}) .
$$

Let $k:=-\lambda-2$. If $\lambda$ is an integer and if $\lambda \leq-2$ (i.e. $k$ is a nonnegative integer), then the partial derivative of $f$ with respect to the coordinate $x^{i}$ should be calculated by means of the formula (cf. equation (5.15) in the paper of Kopeikin [5])

$$
\partial_{i} f=\left(\partial_{i} f\right)_{\text {ord }}+\frac{(-1)^{k}}{k !} \frac{\partial^{k} \delta(\boldsymbol{x})}{\partial x^{i_{1}} \ldots \partial x^{i_{k}}} \oint_{\Sigma} d S_{i} f x^{i_{1}} \ldots x^{i_{k}}
$$

where $\left(\partial_{i} f\right)_{\text {ord }}$ means the derivative computed using the standard rules of differentiations, $\Sigma$ is any smooth close surface surrounding the origin and $d S_{i}$ is the surface element on $\Sigma$.

EXAMPLE 4.1. Let us employ formula (24) to calculate $\partial_{i} \partial_{j} \frac{1}{r}$. The result is

$$
\partial_{i} \partial_{j} \frac{1}{r}=\left(3 n^{i} n^{j}-\delta_{i j}\right) \frac{1}{r^{3}}-\frac{4 \pi}{3} \delta_{i j} \delta(\boldsymbol{x}) .
$$

From the above it follows that $\Delta \frac{1}{r}=-4 \pi \delta(\boldsymbol{x})$.

5. The compatibility problem. It turns out that one can use several different procedures to regularize one divergent integral. The natural question arises about the compatibility of the results obtained by means of different methods. Here we show an example which illustrates the problem.

EXAMPLE 5.1. Let us consider the following divergent integral $(a \neq b)$ :

$$
n_{a b}^{i} n_{a b}^{j} \int d^{3} x\left(\partial_{i} \partial_{j} \frac{1}{r_{a}}\right) \frac{1}{r_{b}^{4}} .
$$

We shall regularize this integral in two different ways. We first replace in (26) differentiations with respect to $x^{i}$ by those with respect to $x_{a}^{i}$, which we denote by $\partial_{i}^{(a)}$ (obviously $\left.\partial_{i} r_{a}=-\partial_{i}^{(a)} r_{a}\right)$. Then we shift the differentiations before the integral sign and apply directly definition (13). The result is

$$
\begin{aligned}
{\left[n_{a b}^{i} n_{a b}^{j} \int d^{3} x\left(\partial_{i} \partial_{j} \frac{1}{r_{a}}\right) \frac{1}{r_{b}^{4}}\right]_{\mathrm{reg}} } & =n_{a b}^{i} n_{a b}^{j} \partial_{i}^{(a)} \partial_{j}^{(a)}\left[\int d^{3} x \frac{1}{r_{a} r_{b}^{4}}\right]_{\mathrm{reg}}= \\
& =n_{a b}^{i} n_{a b}^{j} \partial_{i}^{(a)} \partial_{j}^{(a)}\left(-\frac{2 \pi}{r_{a b}^{2}}\right)=-\frac{12 \pi}{r_{a b}^{4}}
\end{aligned}
$$

We obtained the result (27) performing integration first and then differentiation. Now we shall regularize the integral (26) doing differentiation first. To do it we have to use the rule (24), which gives (cf. (25))

$$
\partial_{i} \partial_{j} \frac{1}{r_{a}}=\left(3 n_{a}^{i} n_{a}^{j}-\delta_{i j}\right) \frac{1}{r_{a}^{3}}-\frac{4 \pi}{3} \delta_{i j} \delta\left(\boldsymbol{x}-\boldsymbol{x}_{a}\right) .
$$

We substitute (28) into (26):

$$
\left[n_{a b}^{i} n_{a b}^{j} \int d^{3} x\left(\partial_{i} \partial_{j} \frac{1}{r_{a}}\right) \frac{1}{r_{b}^{4}}\right]_{\mathrm{reg}}=\left[\int d^{3} x \frac{3\left(\boldsymbol{n}_{a} \cdot \boldsymbol{n}_{a b}\right)^{2}-1}{r_{a}^{3} r_{b}^{4}}\right]_{\mathrm{reg}}-\frac{4 \pi}{3} \int d^{3} x \frac{\delta\left(\boldsymbol{x}-\boldsymbol{x}_{a}\right)}{r_{b}^{4}} .
$$


The second integral on the right-hand side of (29) can be calculated by means of definition (4). The result is

$$
-\frac{4 \pi}{3} \int d^{3} x \frac{\delta\left(\boldsymbol{x}-\boldsymbol{x}_{a}\right)}{r_{b}^{4}}=-\frac{4 \pi}{3 r_{a b}^{4}} .
$$

To calculate the first integral on the right-hand side of (29) we use first the relationship

$$
\boldsymbol{n}_{a} \cdot \boldsymbol{n}_{a b}=\frac{r_{b}^{2}-r_{a}^{2}-r_{a b}^{2}}{2 r_{a} r_{a b}}
$$

which enables us to put the integrand in the form (19). Then we apply definition (22). We obtain

$$
\left[\int d^{3} x \frac{3\left(\boldsymbol{n}_{a} \cdot \boldsymbol{n}_{a b}\right)^{2}-1}{r_{a}^{3} r_{b}^{4}}\right]_{\mathrm{reg}}=-\frac{32 \pi}{3 r_{a b}^{4}} .
$$

Collecting (30) and (32) together we finally obtain

$$
\left[n_{a b}^{i} n_{a b}^{j} \int d^{3} x\left(\partial_{i} \partial_{j} \frac{1}{r_{a}}\right) \frac{1}{r_{b}^{4}}\right]_{\mathrm{reg}}=-\frac{12 \pi}{r_{a b}^{4}}
$$

which coincides with the result (27) obtained before. The two ways of regularizing the integral (26), described above, coincide if and only if we apply formula (24) when we perform differentiation before integration.

6. Integrals of more general type. Let us now restrict ourselves, for simplicity, to the two body system, i.e. $a, b, \ldots \in\{1,2\}$. A typical integrand in the studying of the 3rd post-Newtonian Hamilton's function for the two-body system has the form

$$
f=\sum_{i=1}^{N} c_{J_{i 1} \ldots J_{i j_{i}}} r_{1}^{\alpha_{i}} r_{2}^{\beta_{i}}\left(\partial_{K_{i 1}} \ldots \partial_{K_{i k_{i}}} r_{1}\right)\left(\partial_{L_{i 1}} \ldots \partial_{L_{i l_{i}}} r_{1}\right) \ldots\left(\partial_{M_{i 1}} \ldots \partial_{M_{i m_{i}}} r_{2}\right) \ldots
$$

where $c_{J_{i 1} \ldots J_{i j_{i}}}, \alpha_{i}$, and $\beta_{i}$ are constants. We want to regularize the integral of $f$ which is typically divergent. To do this we could use definition (24) of differentiation of homogeneous functions for each derivative in (34) separately, but then we would obtain products of identical Dirac delta distributions. To avoid this we shall proceed differently.

We first perform all differentiations in (34) employing the standard rules but keeping scalar products of identical unit vectors (i.e. we do not put $\boldsymbol{n}_{a} \cdot \boldsymbol{n}_{a}=1$ ). Importance of this point will be illustrated in the next example. After this operation the integrand takes the form

$$
f=\sum_{i=1}^{N^{\prime}} c_{J_{i 1}^{\prime} \ldots J_{i j_{i}}^{\prime}}\left(n_{1}^{K_{i 1}^{\prime}} \ldots n_{1}^{K_{i k_{i}}^{\prime}} r_{1}^{\alpha_{i}^{\prime}}\right)\left(n_{2}^{L_{i 1}^{\prime}} \ldots n_{2}^{L_{i l_{i}}^{\prime}} r_{2}^{\beta_{i}^{\prime}}\right)
$$

Next we use a set of formulae to replace products of unit vectors by combinations of radii and their derivatives. Here we show only three simplest relations of this type:

$$
\begin{aligned}
r_{a}^{\alpha} n_{a}^{i} & =\frac{\partial_{i} r_{a}^{\alpha+1}}{\alpha+1}, \\
r_{a}^{\alpha} n_{a}^{i} n_{a}^{j} & =-\frac{\delta_{i j} r_{a}^{\alpha}}{\alpha}+\frac{\partial_{i} \partial_{j} r_{a}^{\alpha+2}}{\alpha(\alpha+2)},
\end{aligned}
$$




$$
r_{a}^{\alpha} n_{a}^{i} n_{a}^{j} n_{a}^{k}=-\frac{\left(\delta_{i j} \partial_{k}+\delta_{i k} \partial_{j}+\delta_{j k} \partial_{i}\right) r_{a}^{\alpha+1}}{(\alpha-1)(\alpha+1)}+\frac{\partial_{i} \partial_{j} \partial_{k} r_{a}^{\alpha+3}}{(\alpha-1)(\alpha+1)(\alpha+3)}
$$

Now the integrand looks as follows

$$
f=\sum_{i=1}^{N^{\prime \prime}} c_{J_{i 1}^{\prime \prime} \ldots J_{i j_{i}}^{\prime \prime}}\left(\partial_{K_{i 1}^{\prime \prime}} \ldots \partial_{K_{i k_{i}}^{\prime \prime}} r_{1}^{\alpha_{i}^{\prime \prime}}\right)\left(\partial_{L_{i 1}^{\prime \prime}} \ldots \partial_{L_{i l_{i}}^{\prime \prime}} r_{2}^{\beta_{i}^{\prime \prime}}\right) .
$$

Using the representation (36) we can calculate the integral of $f$ in two different ways, analogously to what was done in Example 5.1. Firstly, to regularize the integral over $f$ we shift all differentiations before integral signs and then we use formula (13) for individual integrands. More precisely, we calculate the limit

$$
\begin{aligned}
\lim _{\varepsilon \rightarrow 0}\left[\sum_{i=1}^{N^{\prime \prime}}(-1)^{k_{i}+l_{i}} c_{J_{i 1}^{\prime \prime} \ldots J_{i j_{i}}^{\prime \prime}} R\left(\alpha_{i}^{\prime \prime}+\mu \varepsilon, \beta_{i}^{\prime \prime}+\nu \varepsilon\right)\right. \\
\\
\left.\quad \times \partial_{K_{i 1}^{\prime \prime}}^{(1)} \ldots \partial_{K_{i k_{i}}^{\prime \prime}}^{(1)} \partial_{L_{i 1}^{\prime \prime}}^{(2)} \ldots \partial_{L_{i i_{i}}^{\prime \prime}}^{(2)} r_{12}^{\alpha_{i}^{\prime \prime}+\beta_{i}^{\prime \prime}+(\mu+\nu) \varepsilon+3}\right] .
\end{aligned}
$$

It turns out again that it is enough to consider only limits which have the form (17) and again as a regularized value of the integral of $f$ we take, by definition, the number $A$ of (17). Secondly, we can perform all differentiations in (36) before integration. To do this we must use the enhanced rule of differentiation (24). After this the integrand will be a sum of two parts: the first part will contain no Dirac delta distributions, whereas the second one will contain some. The first part will be of type showed in (19) and to calculate the integral over it we shall use definition (22). The second part is calculated by means of formula (4). We have observed that at the 3rd post-Newtonian order both ways give the same result.

In the next example we compare the two just described equivalent methods of regularizing with the Hadamard's "partie finie" regularization from Section 2.

ExAMPLE 6.1. The Newtonian gravitational potential which describes the system of two point particles of masses $m_{1}$ and $m_{2}$ is, up to a constant, equal to

$$
\phi=\frac{m_{1}}{r_{1}}+\frac{m_{2}}{r_{2}} .
$$

At the $n-1$ post-Newtonian order we have to compute the following integral

$$
\int d^{3} x \phi^{n}(\Delta \phi) \text {. }
$$

In deriving Hamilton's function we assume that surface terms obtained during integration by parts can always be neglected. So (39) can, with the aid of integration by parts, be put into the form

$$
-n \int d^{3} x \phi^{n-1}(\nabla \phi)^{2}
$$

Because $\Delta \phi=-4 \pi\left(\delta\left(\boldsymbol{x}-\boldsymbol{x}_{1}\right)+\delta\left(\boldsymbol{x}-\boldsymbol{x}_{2}\right)\right)$, we define the first way of computing the integral (39)

$$
\operatorname{Reg}^{(\mathrm{I})}(n):=-4 \pi \int d^{3} x \phi^{n}\left(\delta\left(\boldsymbol{x}-\boldsymbol{x}_{1}\right)+\delta\left(\boldsymbol{x}-\boldsymbol{x}_{2}\right)\right)
$$


where the right-hand side of (41) is computed by means of equation (4). To compute the integral (40) we can use one of the two regularization procedures described below equation (36), so we define

$$
\operatorname{Reg}^{(\mathrm{II})}(n):=-n\left[\int d^{3} x \phi^{n-1}(\nabla \phi)^{2}\right]_{\mathrm{reg}},
$$

where the subscript "reg" means here use one of the two methods. Direct calculation shows that for $n \leq 8$ we have:

$$
\begin{aligned}
& \operatorname{Reg}^{(\mathrm{I})}(n)=\operatorname{Reg}^{(\mathrm{II})}(n) \quad \text { for } n \in\{1,2,3,4,5\} ; \\
& \operatorname{Reg}^{(\mathrm{I})}(n) \neq \operatorname{Reg}^{(\mathrm{II})}(n) \quad \text { for } n \in\{6,7,8\} .
\end{aligned}
$$

It follows that the two methods of regularization give the same results for $n \leq 5$, i.e. up to the 4 th post-Newtonian order. If in (35) we would replace scalar products $\boldsymbol{n}_{a} \cdot \boldsymbol{n}_{a}$ by 1 , then disagreement between the two ways of regularization would be already present for $n=4$, i.e. at the 3rd post-Newtonian order.

Acknowledgements. The author gratefully acknowledges many valuable and stimulating discussions with Gerhard Schäfer as well as his critical reading of the manuscript. The author also thanks Andrzej Królak for useful comments.

\section{References}

[1] R. Arnowitt, S. Deser, and C. W. Misner, The dynamics of general relativity, in: Gravitation: an introduction to current research, L. Witten (ed.), Wiley, New York, 1962, 227-265.

[2] I. M. Gel'fand and G. E. Shilov, Generalized functions, Academic Press, New York, 1964.

[3] P. Jaranowski and G. Schäfer, Radiative 3.5 post-Newtonian ADM Hamiltonian for many-body point-mass systems, Phys. Rev. D (1996), submitted.

[4] P. Jaranowski and G. Schäfer, 3rd post-Newtonian ADM Hamiltonian for two-body pointmass systems, in preparation.

[5] S. M. Kopeikin, General-relativistic equations of binary motion for extended bodies with conservative corrections and radiation damping, Sov. Astron. 29 (1985), 516-524.

[6] T. Ohta, H. Okamura, T. Kimura, and K. Hiida, Higher order gravitational potential for many-body system, Progr. Theor. Phys. 51 (1974), 1220-1238.

[7] M. Riesz, L'intégrale de Riemann-Liouville et le problème de Cauchy, Acta Mathematica 81 (1949), 1-223.

[8] G. Schäfer, The gravitational quadrupole radiation-reaction force and the canonical formalism of ADM, Annals of Physics 161 (1985), 81-100. 